\title{
EXPLORACIÓN PRELIMINAR DEL APRENDIZAJE COLABORATIVO DENTRO UN ENTORNO VIRTUAL
}

PRELIMINARY STUDY OF COLLABORATIVE LEARNING IN A VIRTUAL ENVIRONMENT

\section{Volumen 12, Número 3 \\ Setiembre-Diciembre}

pp. 1-20

Este número se publicó el 30 de setiembre de 2012

Gilles Lavigne

María Pricila Vasconcelos Ovando Javier Organista Sandoval

Lewis McAnally Salas

Revista indizada en $\underline{\text { REDALYC }}$

Revista distribuida en las bases de datos:

CATÁLOGO DE LATINDEX, IRESIE, CLASE, DIALNET, DOAJ, E-REVIST@S,

Revista registrada en los directorios:

ULRICH'S, REDIE, RINACE, OEI, MAESTROTECA, PREAL, CLASCO 


\title{
EXPLORACIÓN PRELIMINAR DEL APRENDIZAJE COLABORATIVO DENTRO UN ENTORNO VIRTUAL \\ PRELIMINARY STUDY OF COLLABORATIVE LEARNING IN A VIRTUAL ENVIRONMENT
}

\author{
Gilles Lavigne ${ }^{1}$ \\ María Pricila Vasconcelos Ovando ${ }^{2}$ \\ Javier Organista Sandoval ${ }^{3}$ \\ Lewis McAnally Salas ${ }^{4}$
}

Resumen: En este artículo se presenta el resultado de una investigación cuyo objeto de estudio fue la utilidad del uso de foros para la construcción del conocimiento en el aprendizaje colaborativo virtual; a la fecha, es poco lo que se conoce sobre las prácticas de navegación de los estudiantes en el espacio virtual. Se analizaron los registros de navegación (logs) en la plataforma Moodle utilizada por estudiantes al nivel de Maestría en la Universidad Autónoma de Baja California dentro de un curso en el que los foros fueron considerados como una herramienta de aprendizaje. Después del análisis estadístico de los datos se concluye que el uso de los foros puede propiciar el desarrollo del aprendizaje colaborativo. Este trabajo es una primera aproximación sobre lo que sucede alrededor del aprendizaje colaborativo virtual.

Palabras clave: MOODLE, FORO, APRENDIZAJE COLABORATIVO VIRTUAL, NAVEGACIÓN, LOGS, MÉXICO

Abstract: This article shows up the results of an investigation about the use of forums for the construction of the knowledge in a collaborative virtual learning environment; to date it is little what is known on students' surfing practices in a virtual learning space. This study analyzes the logs registered in the Moodle platform as the whereabouts of Postgraduate students of the Universidad Autónoma de Baja California within a course in which forums were considered as a learning tool. The statistical analysis of the data leads to the conclusion that the use of forums can promote the development of collaborative learning. This work is a first approach to understand and to know what happens within virtual collaborative learning environments.

Key words: MOODLE, FORUM, COLLABORATIVE ELEARNING, NAVIGATION, LOGS, MEXICO

\footnotetext{
1 Investigador, Instituto de Investigación y Desarrollo Educativo, Universidad Autónoma de Baja California, México. Dirección electrónica: gilles@uabc.edu.mx

2 Coordinadora, Coordinación de Educación a Distancia, Universidad Tecnológica de Tabasco, México.Dirección electrónica: pricila15@hotmail.com

3 Instituto de Investigación y Desarrollo Educativo, Universidad Autónoma de Baja California, México. Dirección electrónica: javor@uabc.edu.mx

4 Investigador, Instituto de Investigación y Desarrollo Educativo, Universidad Autónoma de Baja California, México. Dirección electrónica: mcanally@uabc.edu.mx
}

Artículo recibido: 8 de febrero, 2012 Aprobado: 6 de agosto, 2012 


\section{Introducción a la problemática}

La Internet está propiciando el surgimiento y la consolidación de nuevas estructuras sociales y formas de organización en las que las referencias espacio-temporales tradicionales no tienen validez, y que se producen en un espacio cibernético virtual. La constitución gradual de la sociedad como nueva estructura social es expresión de esta transformación para convertirnos en la era de la información (Garrido, 2003; Duart, Gil, y Pujol, 2008).

Se focaliza el presente estudio sobre los intercambios de información entre estudiantes en un ámbito virtual de aprendizaje. Las teorías del aprendizaje consideran la importancia de las interacciones sociales entre las personas como proceso de aprendizaje basado en la colaboración. Esta se ha ido incrementando considerablemente con el uso de las Tecnologías de la Información y Comunicación (TIC) centrado en la incorporación de características funcionales, de herramientas tales como chat, foros, correos y blogs que promueven las interacciones. Desde hace una docena de años, las TIC han propuesto una nueva tecnología integradora, dedicada específicamente a la educación, las plataformas ${ }^{5}$ las cuales han sido una de las herramientas tecnológicas que, debido a sus características, han contribuido al fomento de la colaboración entre sus usuarios.

La presente investigación tiene como objetivo explorar si los estudiantes utilizan para aprender la herramienta del foro dentro el espacio virtual de un curso en línea soportado por Moodle, la cual tiene sus propios registros (logs) de navegación. Esos registros permiten identificar cómo los estudiantes utilizan los recursos de comunicación disponibles. Se ha considerado como objeto de estudio en este trabajo la forma de transmisión de la información con el uso de tecnologías y su relación con el aprendizaje colaborativo. La colaboración en el aprendizaje se ha ido incrementando considerablemente; en este sentido, un fenómeno asociado al aprendizaje con el uso de las TIC se centra en la incorporación de características funcionales de herramientas como foros que promueven el aprendizaje colaborativo, pero se necesita tener los conocimientos necesarios de cómo y porqué los estudiantes utilizan los medios de comunicación en el ambiente virtual, para tener la habilidad para aplicarlos efectivamente. En este texto se da a conocer la forma en que los

${ }^{5}$ El lanzamiento del primer sistema de entorno de aprendizaje virtual, WebCT, ocurrió en 1997, y el de Moodle en 2002. 
estudiantes de posgrados utilizaron los foros de la plataforma Moodle dentro un curso híbrido o bimodal en la Universidad Autónoma de Baja California.

\section{Aprendizaje colaborativo}

La colaboración en el aprendizaje no es un tema nuevo; sin embargo, es con la remodelación de la pedagogía en el siglo XX y el desarrollo de las TIC que se regeneraron la reflexión y la práctica acerca el aprendizaje colaborativo. Las primeras ideas de colaboración en el aprendizaje se vieron reflejadas con las aportaciones de Dewey (1989). En la misma época, el soviético Vygotsky postula la importancia del aprendizaje en un ambiente propicio para el alumno, y considera al individuo como el resultado del proceso histórico y social donde el conocimiento es un proceso de interacción entre el sujeto y el medio, entendido como algo social y cultural, no solamente físico (Ríos, 2005). Además, surgen las reflexiones de Piaget acerca del proceso de aprendizaje que se le va otorgando a la persona que aprende un rol activo en la construcción del conocimiento (Sacristán y Pérez, 1994).

Para Vygotsky, los nuevos conocimientos se forman a partir de los propios esquemas de la persona, producto de su realidad y son comparados con los esquemas de los demás individuos que lo rodean (Garcia, 2000). Su concepto de Zona de Desarrollo Próximo (ZDP) se define como la distancia entre el nivel de desarrollo, determinado por la capacidad de resolver independientemente un problema, y el nivel de desarrollo potencial, determinado a través de la resolución de un problema bajo la guía de una persona experta, o en colaboración con otro compañero más capaz (Vygotsky, 2005). Desde el inicio de la reflexión acerca la nueva pedagogía en la primera mitad del siglo XX la problemática del aprendizaje colaborativo es planteado.

Para comprender mejor el objeto de esta investigación, es necesario hacer una escala para definir y acentuar los términos que están inmiscuidos en ella: trabajo en equipo, cooperación y colaboración. Para la Real Academia Española, un equipo es un grupo de personas organizado para una investigación o servicio determinado, y define colaborar como: trabajar con otra u otras personas en la realización de una obra, y cooperar como: obrar juntamente con otro u otros para un mismo fin. Es decir, los términos refieren a algo similar, incluso se menciona el término cooperar como sinónimo de colaborar.

Aunque existen infinidad de autores que, por un lado, separan y, por otro lado, unen al aprendizaje colaborativo y cooperativo, el presente trabajo considera ambos enfoques, tal 
como lo menciona Slavin (citado en Crook, 1998), como complementarios. Este trabajo se sitúa con el grupo de autores que consideran similares los dos enfoques y para términos de homogeneidad se utilizará en adelante solo el término aprendizaje colaborativo.

El aprendizaje colaborativo, tal y como ahora se conoce, empieza a surgir a principios de los años 1970. En esa época destacaron los trabajos de Johnson y Johnson (1989) en los Estados Unidos, quienes empezaron a desarrollar el aprender juntos, al igual que en España Serrano y Calvo (1994) trabajaron sobre la dicotomía cooperación/competición. Algunas de las investigaciones que surgieron fueron trabajos relacionados con la socialización y la propia adquisición de competencias sociales. Otros estudios, además, se centran en el desarrollo cognitivo y social que permiten la transmisión de información, la cooperación y la solución constructiva de conflictos (Pujolas, 2004). Por fin se debe entender que el aprendizaje colaborativo resulta de un trabajo hecho junto, en colaboración o cooperación.

El trabajo conjunto entre pares, entre novatos y expertos hace evidente que la interacción social trae beneficios al aprendizaje. Muhlenbrock (1999) considera que el aprendizaje colaborativo es el compromiso mutuo establecido entre personas en un grupo, que se unen en un esfuerzo coordinado para dar respuesta a una tarea. Para él, este tipo de organización permite entender los procesos que se gestan al trabajar entre pares. El trabajo colaborativo persigue una mejora de las propias relaciones sociales (Crook, 1998). Es decir, en el enfoque colaborativo de organización del trabajo se considera esencial la interacción alumno-profesor pero también la interacción alumno-alumno, los alumnos aprendiendo unos de otros, así como de su profesor y del entorno (Glinz, 2005).

Para Brown y Atkins (citado en Escribano, 1995) los objetivos que persigue el aprendizaje colaborativo se centran en el desarrollo de estrategias de comprensión y explicación, de preguntas y respuestas. La discusión y el debate sirven, en primer lugar, para desarrollar las habilidades de comunicación con otros y la utilización precisa del lenguaje. En segundo lugar, genera el desarrollo de competencias intelectuales y profesionales, como por ejemplo: analizar, razonar, pensar críticamente, sintetizar, diseñar, etc. Y, por último, el aprendizaje colaborativo también promueve el crecimiento personal de los estudiantes, que incluye el desarrollo de estrategias de comunicación y pensamiento, el desarrollo de la autoestima, dirige el propio aprendizaje, aprende a trabajar con otros y a conocerse a sí mismo y a los demás. 
Por lo tanto, el aprendizaje colaborativo es una estrategia eficaz para conseguir objetivos de orden intelectual y social, es decir, los grupos no pueden funcionar sin habilidades sociales enfocadas a la toma de decisiones, construcción de ideas, comunicación y resolución de conflictos. Por eso, estas habilidades se deben desarrollar a la vez y con la misma precisión que las habilidades académicas (Alvarez, Ayuste, Gros, Guerra, y Romaña, 2005).

En resumen, se puede considerar realmente colaborativo un grupo cuando integra al menos: la interdependencia positiva, la interacción, la responsabilidad individual, y el uso de habilidades interpersonales y grupales por parte de los miembros del grupo (Brito, 2004). Entonces, el trabajo colaborativo hace necesaria la construcción de conocimientos (Calzadilla, 2002).

\section{Aprendizaje colaborativo virtual}

El aprendizaje colaborativo es caracterizado por la interacción y el aporte de todos en la construcción del conocimiento y necesario para el logro de metas académicas, ya que facilita la comprensión y la explicación de temas por los propios compañeros, fortaleciendo así las relaciones sociales entre los mismos (Brito, 2004). Así, se forma una colectividad no competitiva, en la cual todos los miembros colaboran y contribuyen al aprendizaje de todos. Y para hacerlo, cada miembro del grupo comparte experiencias y conocimientos con base en una meta específica (Bernaza y Lee, 2005).

Con el apoyo de las TIC, las situaciones de aprendizaje son más variadas y sobre todo cambian las situaciones de aprendizaje tradicionales enfocadas a un tiempo y un lugar. Autores como Bereiter y Engeström (citado en Liponen, Hakkarainen, y Paavola, 2004) han estudiado cómo una comunidad es capaz de transformar, superar y expandir sus logros a través de actividades colaborativas, fortaleciendo estos logros a través de herramientas representacionales como el lenguaje, ideas, modelos y en este caso también las TIC. El papel de las TIC es facilitar la comunicación, pero también ser gestor y organizador para dar fundamento al trabajo de un grupo de personas en tareas de aprendizaje; además, cuando existe la posibilidad de registrar los procesos de trabajo, se pueden establecer modelos que permiten analizarlos, monitorearlos o en su caso intervenir para mejorarlos (Barros y Verdejo, 2001). 
Desde el punto de vista pedagógico, las TIC representan ventajas para el proceso de aprendizaje colaborativo, en cuanto a cinco características que se pueden señalar: estimular la comunicación interpersonal, facilitan el trabajo colaborativo, seguir el progreso del grupo, a nivel individual y colectivo, acceder a información y contenidos de aprendizaje, crear ejercicios de evaluación y autoevaluación (Calzadilla, 2002, p. 8). Este tipo de aprendizaje aporta beneficios en el aprendizaje de los estudiantes que pueden resumirse en: habilidades de pensamiento crítico, autonomía en el aprendizaje, colaboraciones más efectivas y habilidades sociales, personales y de grupo (Cabrera, 2005, p. 3). Conocer las prácticas de navegación de los estudiantes facilitará la comprensión de sus aprendizajes colaborativos en el espacio virtual.

En otras palabras, este tipo de aprendizaje no solo origina cambios en la forma como se estructura la organización escolar, sino que también aporta beneficios en el aprendizaje de los estudiantes que pueden resumirse en: habilidades de pensamiento crítico, autonomía en el aprendizaje, colaboraciones más efectivas y habilidades sociales, personales y de grupo (Cabrera, 2005).

El aprendizaje colaborativo en línea hace énfasis en la estructura de aprendizaje centrado en el estudiante, resaltando las actividades a realizar sobre los contenidos a transmitir; donde la tecnología es interpretada como una herramienta mediadora denominada: entorno virtual de aprendizaje. Aquí, el sistema de organización y presentación de la información facilita el papel activo del usuario, escogiendo los trayectos de su exploración y proporcionando el acceso al contenido de forma dinámica (Duart, Gil, Pujol, y Castaño, 2008). Es decir, impulsa en el alumno un proceso de decisión al proporcionarle libertad de elección de movimientos en su inmersión al sistema, además de la posibilidad que el alumno pueda relacionarse con otras personas que también accedan a él.

Dentro de las innovaciones tecnológicas aportadas al ámbito escolar destaca la educación en línea. Este tipo de educación, aún cuando suele considerarse parte esencial de la educación a distancia, también es utilizado como complemento de la educación presencial (Martí, 1992), la cual se califica entonces de híbrida o bimodal (Lavigne, Backhoff, y Organista, 2008). Las TIC se presentan como un medio eficaz para avanzar hacia una redefinición curricular que busca, sobre todo, proveer de las estrategias de análisis y resolución de problemas indispensables hoy en día para los alumnos (Bartolomé, 2002). 
Pero no hay muchas publicaciones, por lo pronto, de cómo los estudiantes utilizan las herramientas de aprendizaje colaborativo disponibles en línea, ni de cómo navegan en el espacio virtual de aprendizaje ${ }^{6}$. Aunque los cursos diseñados para el aprendizaje en línea tienen rutas de aprendizaje elaboradas por los profesores que han de seguir los estudiantes, no es seguro que lo hagan de la forma prescrita. Pero existe la posibilidad de estudiar la navegación de estudiantes para identificar sus prácticas de participación, por ejemplo, en los foros, a través de sus movimientos en el entorno virtual.

De hecho, no se sabe lo que pasa en estos entornos virtuales, pero sí, se sabe que gracias a la estructura del web y al hecho que los servidores donde están hospedados los sistemas de aprendizaje virtual tienen programas para registrar todos los movimientos realizados por los usuarios, se pueden seguir los movimientos de una página a otra dentro un mismo servidor, o de un servidor a otro; es lo que se llama Web Surfing (Polly, 1992). Sin embargo, el análisis de estos registros se enfrenta a varios problemas técnicos, debido a la diversidad, al tamaño y a la organización de las bases de datos; también a problemas metodológicos asociados tanto a la identificación de características propias y únicas, como a la generalización. Este tipo de análisis se llama Minería de Datos, y cuando se aplica al campo de la educación, Minería de Datos Educativa (Educational Data Mining) (IWGEDM, 2011; Baker y Yacef, 2011).

Hasta ahora, los estudios de minería de datos educativa, como campo dedicado a la investigación de la educación en línea, se realizaron principalmente para construir modelos de las características de los estudiantes, para mejorar las estructuras de los cursos, para fortalecer el soporte tutorial a los estudiantes y buscar relaciones con fines de prever o de agrupar (Baker y Yacef, 2011). Según Hosking y van Hooff (2005), poco se conoce acerca del impacto sobre el logro académico del estudiante. A partir de una investigación realizada en la Universidad de Portsmouth, donde exploraron la posible influencia pedagógica del uso de un sitio Web (hits, cantidad y duración de accesos, mensajes enviados o publicados, etc.) los resultados mostraron las bondades educativas del diálogo por medios electrónicos, específicamente en la impartición de asesorías y la aclaración de dudas.

\footnotetext{
${ }^{6}$ Se puede consultar el sitio de la Fundación Wikimedia, con la rúbrica "Aprendizaje colaborativo, Referencias", se cuentan decenas de referencias, pero ninguna acerca de cómo navegan los estudiantes dentro los entornos virtuales.
} http://es.wikibooks.org/wiki/Aprendizaje colaborativo/Referencias 
Se encuentran muy pocos artículos vinculados con el aprendizaje colaborativo. Así, Dringus y Ellis, (2005) indagaron con la web minería cuál era la estructura de navegación de los estudiantes a partir de los textos agregados en los foros. Dönmez, Rosé, Stegmann, Weinberger, y Fischer, (2005) utilizaron también la web minería para realizar un análisis multidimensional del aprendizaje colaborativo a través el tratamiento lingüístico del contenido de foros, para desarrollar una tecnología de análisis automática. Este método permitió también revelar las operaciones de grupos de trabajo colaborativo para ayudar a seguir los avances de las tareas (Perera, Kay, Koprinska, Yacef, y Zaiane, 2009).

Sin embargo, no es una obligación aplicar las técnicas de la minería de datos para analizar los registros de navegación con fines educativos. Los sistemas de administración de cursos en línea, como Moodle o Blackboard, tienen sus propios programas para registrar los ingresos al sistema y las actividades realizadas en el espacio virtual por parte de cada estudiante; se presenta, entonces, la posibilidad de recuperar esta información para su procesamiento y análisis. De hecho, existen algunos estudios acerca los registros de navegación en entornos virtuales educativos sin la aplicación de las técnicas de la minería de datos. Los estudios preliminares realizados por Lavigne y sus colegas han establecido un procedimiento metodológico y algunos resultados de referencia (Lavigne, Organista, y McAnally, 2008; Lavigne, McAnally, Organista, Días, y Vasconcelos, 2011; Días, 2010; Vasconcelos, 2011).

Además, el estudio de los logs se desarrolla también al margen de la Minería de datos educativa, con el concepto de Learning Analytics, que se puede traducir como el "análisis lógico de los datos de aprendizaje del web"; la empresa Solar (Society for Learning Analytics Research) define este concepto metodológico como: the measurement, collection, analysis and reporting of data about learners and their contexts, for purposes of understanding and optimizing learning and the environments in which it occurs http://www.solaresearch.org/mission/about/ (SOLAR, 2011).

\section{Método}

La investigación es fue de tipo exploratorio, su diseño tuvo dos componentes:

1. Extraer de Moodle los registros de navegación para analizar cómo los estudiantes utilizan la herramienta del foro. 
2. Obtener y analizar las opiniones y percepciones de los estudiantes acerca Moodle y el aprendizaje colaborativo mediante un cuestionario y grupos de discusión.

En el presente artículo se destacan solamente los resultados del primer componente.

\subsection{Participantes y entorno}

El universo de estudio está conformado por 23 alumnos de la cohorte 2008-2010 de la Maestría en Ciencias Educativas del IIDE, específicamente quienes cursaron la asignatura de Planeación y Evaluación Educativa (PEE) en línea en el cuatrimestre enero-abril 2009.

Esta materia fue impartida en línea con Moodle a través del Sistema @ulas-UABC. En el curso se contaron 10 foros, 4 generales y 6 temáticos, pero solo se tomaron en cuenta, en este estudio, los foros académicos, es decir, los foros temáticos:

- Foro 1 El contexto internacional: donde analizaron las políticas y posiciones de organismos internacionales como la OCDE y el Banco Mundial en relación con la educación.

- Foro 2 El ámbito nacional: en este foro analizaron las políticas y posiciones que la SEP promueve para las instituciones de educación superior del país.

- Foro 3 Planeación estratégica: en este foro el alumno, para poder participar, primero debía investigar en la Internet tres sitios acerca del tema, justificar por qué los escogió y explicar el contenido de los sitios encontrados.

- Foro 4 Planeación prospectiva: este foro se realizó bajo la misma dinámica del anterior, explicando y justificando los sitios encontrados acerca del tema.

- Foro 5 Presentación del tema de Glazman y diálogo: para este foro se realizó por equipo una presentación en PowerPoint(C, derivada de las lecturas correspondientes al tema y fueron subidas al foro para su debate y explicación.

- Foro 6 Análisis y alternativas de evaluación: en este foro se trabajó en equipo seleccionando una materia de alguna de las carreras que ofrece la UABC, para analizar los mecanismos de evaluación que el profesor ejerce, el resultado de este trabajo se presentó en este foro para ser analizado y debatido por el grupo. 


\subsection{Instrumentos: registros de navegación}

Un registro de servidor (server log) es un archivo, o varios, creado y mantenido por un servidor web de forma automática de la actividad realizada por el mismo; tal servidor mantiene un historial de las peticiones de página por los usuarios, formado con la información que se agrega normalmente con la solicitud, incluyendo dirección IP del cliente, fecha de la solicitud/tiempo, página que solicita, código HTTP, bytes comunicados, agente de usuario (protocolos), y remitente (referrer URL). ${ }^{7}$ La plataforma Moodle crea sus propios registros de actividad en línea registrando todos los movimientos de los participantes, especificando el recurso o espacio donde ha estado el alumno, las veces que ha estado y el tiempo que estuvo en cada espacio. En resumen, los registros de actividad en línea se crean con cada clic que el alumno realiza dentro de la plataforma.

La noción de clic, tal como es utilizado en esta investigación, se entiende como el elemento a través de cual se puede describir y medir las acciones de los estudiantes en un espacio virtual de aprendizaje. En la Internet, un clic corresponde a la presión sobre el botón primario del ratón con el cual se abre una página web o se cambia de página. Se induce en el clic un movimiento dentro del espacio virtual de la Internet de una página a otra. Entonces, el término de clics se puede utilizar en español como equivalente de HITS que en el inglés significa Hypertext Induced Topic Selection. La medición de un clic corresponde, en realidad, a la medición del tiempo entre dos clics. Se extrajeron únicamente los registros de actividad en línea de los foros donde se dialogaron los temas propios del curso.

El sistema Moodle tiene registros asociados con los foros clasificados en cuatro categorías según la acción que el alumno realiza:

- Vista: se refiere al momento en que el alumno entra al foro solo a ver de qué se está hablando, solo entra a dar un vistazo al foro.

- Agregar: este registro se genera cuando el alumno ha generado un comentario nuevo para ser debatido dentro del tema del foro.

- Actualizar: se realiza cuando el alumno aporta a un comentario hecho por otro alumno.

- Borrar: este registro se genera, cuando el alumno agrega un comentario nuevo o realiza una aportación a un comentario generado por alguien más, pero después de anexarlo, por diversas razones lo borra. Esta acción la puede realizar en los siguientes

\footnotetext{
${ }^{7}$ Se puede consultar el sitio de Wikipedia, en inglés, donde se encuentran muchas páginas de explicación de los términos acerca los logs. http://en.wikipedia.org/wiki/Server log
} 
30 minutos después de haberlo hecho, ya que después de ese tiempo, el sistema ya no permite borrar el comentario.

\subsection{Procedimiento}

El proceso de extracción de los datos se realizó de la siguiente manera:

- Se seleccionaron los seis foros académicos con los que contó el curso.

- Se extrajeron de la plataforma Moodle los registros en formato ExcelO.

- Después de extraerlos se hizo la limpieza de los datos, concentrándose específicamente en los registros generados entre el 14 de enero y el 30 de abril de 2009, periodo que comprendió el cuatrimestre.

- Seguido de la limpieza de los datos, se organizó la información de manera que pueda mostrar las actividades que realizó cada alumno en los foros.

- Seguidamente se organizaron los datos por actividad realizada en cada foro, estableciendo el número de clics según la actividad que realizaron los alumnos, ya sea vista, agregar, actualizar o borrar

- De los datos ya organizados se realizaron tablas que muestran las actividades más frecuentes realizadas en los foros de los alumnos que comprenden la muestra y el porcentaje que le corresponde.

\section{Resultados}

Los resultados que se muestran son exclusivamente de los foros académicos obligatorios encontrados en el curso. El foro provee al alumno la facilidad de interactuar con sus compañeros en el diálogo sobre un tema especifico, en este caso temas relacionados con el curso de PEE. El alumno podía ingresar en el foro un comentario relacionado con el tema, para ser leído y debatido por los demás alumnos; asimismo, podía tanto responder un comentario iniciado por otro alumno como proponer un nuevo tema de discusión. Dentro del análisis de los registros de navegación en línea de los estudiantes, se extrajeron los resultados de los clics hechos por los alumnos en toda la navegación que realizaron en el espacio virtual de aprendizaje, así como también el total de clics hechos en los foros con sus actividades específicas (ver tabla $\mathrm{N} . \stackrel{\circ}{1}$ ). A un nivel general, se puede mencionar que la edad de los 23 alumnos oscila entre los 22 y los 46 años, y que las características de género (femenino-masculino) y estado civil (soltero-casado), se encuentran en porcentajes similares. 
Tabla N. 1: Descriptivos básicos del total de clics hechos en el curso

\begin{tabular}{|c|r|r|r|r|r|c|}
\hline $\mathbf{N}=\mathbf{2 6}$ & Mínimo & Máximo & Suma & Media & $\begin{array}{c}\text { Desv. } \\
\text { típ. }\end{array}$ & $\%$ \\
\hline Total Clics & 805 & 5859 & 43546 & 1674.85 & 1013.46 & \\
\hline Foros Total Clics & 312 & 2735 & 23202 & 892.39 & 516,68 & $53.28^{*}$ \\
\hline Agregar Discusión Temas & 2 & 33 & 385 & 14.81 & 7,07 & $1.66^{\star *}$ \\
\hline $\begin{array}{c}\text { Agregar, actualizar, borrar } \\
\text { comentarios }\end{array}$ & 13 & 130 & 1453 & 55.89 & 33.47 & $6.26^{\star *}$ \\
\hline Manejar foros & 0 & 124 & 575 & 22.11 & 25.29 & $2.48^{\star \star}$ \\
\hline Ver discusión temas & 122 & 1089 & 11267 & 433.34 & 280.55 & $48.56^{\star *}$ \\
\hline Ver comentarios & 158 & 1542 & 9522 & 366.23 & 269.31 & $41.04^{\star \star}$ \\
\hline
\end{tabular}

${ }^{*}$ Porcentaje del total de clics en el curso.

**Porcentaje del total de clics en los foros

Se observa que el total de clics realizados en los foros representa la mitad del total de clics realizado en todo el aprendizaje en línea, resaltando la importancia de los foros en este curso. Otra observación es la amplitud de las desviaciones típicas y la de las diferencias entre mínimos y máximos, eso subraya la existencia de prácticas de navegación totalmente en oposición dentro de los estudiantes.

De manera general, se observa que el $90.67 \%$ de clics se encuentra en el área de vista, donde los alumnos solo entraron a ver los comentarios que ya se habían escrito y a analizar desde qué perspectiva se estaba analizando el tema, solo el $7.62 \%$ de los clics corresponde a las aportaciones propias del alumno y un $1.70 \%$ a las aportaciones hechas a los comentarios de otros alumnos (ver tabla $\mathrm{N} . \stackrel{0}{2}$ ). 
Tabla N.․ 2: Distribución de los clics según los foros y las rubricas de Moodle

\begin{tabular}{|c|c|c|c|c|c|c|c|c|c|c|c|}
\hline \multirow{2}{*}{ FOROS } & \multirow{2}{*}{$\mathrm{N}=23$} & \multicolumn{2}{|c|}{ Vista } & \multicolumn{2}{|c|}{ Agregar } & \multicolumn{2}{|c|}{ Actualizar } & \multicolumn{2}{|c|}{ Borrar } & \multicolumn{2}{|c|}{ Total } \\
\hline & & $F$ & $\%$ & $F$ & $\%$ & $F$ & $\%$ & $\mathbf{F}$ & $\%$ & $\mathbf{F}$ & $\%$ \\
\hline \multirow{2}{*}{ Foro 1} & clics & 3152 & 27.83 & 259 & 27.20 & 71 & 36.41 & 3 & 16.66 & 3485 & 27.92 \\
\hline & $\%$ & & 90.44 & & 7.43 & & 2.03 & & 0.08 & & 100.0 \\
\hline \multirow{2}{*}{ Foro 2} & clics & 2680 & 23.67 & 244 & 25.63 & 32 & 16.41 & 1 & 5.55 & 2957 & 23.68 \\
\hline & $\%$ & & 90.63 & & 8.25 & & 1.08 & & 0.03 & & 100 \\
\hline \multirow{2}{*}{ Foro 3} & clics & 1646 & 14.53 & 119 & 12.5 & 29 & 14.87 & 7 & 38.88 & 1801 & 14.43 \\
\hline & $\%$ & & 91.39 & & 6.60 & & 1.6 & & 0.38 & & 100 \\
\hline \multirow{2}{*}{ Foro 4} & clics & 993 & 8.77 & 84 & 8.82 & 24 & 12.30 & 1 & 5.55 & 1102 & 8.82 \\
\hline & $\%$ & & 90.10 & & 7.6 & & 2.17 & & 0.09 & & 100 \\
\hline \multirow{2}{*}{ Foro 5} & clics & 1526 & 13.48 & 121 & 12.71 & 17 & 8.71 & 3 & 16.66 & 1667 & 13.35 \\
\hline & $\%$ & & 91.54 & & 7.25 & & 1.01 & & 0.17 & & 100 \\
\hline \multirow{2}{*}{ Foro 6} & clics & 1325 & 11.71 & 125 & 13.13 & 22 & 11.28 & 3 & 16.66 & 1475 & 11.81 \\
\hline & $\%$ & & 89.83 & & 8.47 & & 1.49 & & 0.20 & & 100 \\
\hline \multicolumn{2}{|c|}{ TOTAL } & 11,322 & 90.67 & 952 & 7.62 & 195 & 1.56 & 18 & 0.14 & $\begin{array}{c}12,48 \\
7\end{array}$ & 100.0 \\
\hline \multicolumn{2}{|c|}{ Promedio* } & 1887 & & 159 & & 33 & & 3 & & 2081 & \\
\hline \multicolumn{2}{|c|}{ Promedio** } & 492 & & 41 & & 8 & & 1 & & 543 & \\
\hline \multicolumn{2}{|c|}{ Promedio $^{\star \star \star}$} & 82 & & 7 & & 1 & & 0 & & 90 & \\
\hline
\end{tabular}

* Promedio por foro

** Promedio por estudiante

*** Promedio por estudiante y por foro

Estos resultados significan que la lectura de los comentarios agregados en los foros es la actividad principal de los estudiantes; eso se puede entender si se toma en cuenta el hecho que cada individuo debe leer los comentarios de los otros estudiantes, lo que requiere muchos más clics que de agregar un nuevo comentario. Se observa que fueron los dos primeros foros que monopolizaron la mitad de todos los clics, en todas las funciones con excepción de Borrar, pero que la necesidad de trabajar por equipo en los dos últimos foros no generó más clics, lo que puede indicar un interés más grande para los temas de los foros iniciales, o, simplemente, subrayar el efecto de la curiosidad asociada a la novedad.

La tabla $\mathrm{N}$. ㅇ 3 muestra cuántas aportaciones (aquí, se trata de la agregación de nuevos temas Forum update post, lo que es diferente de las respuestas Forum add post) fueron generadas por los alumnos en cada foro y cuántas respuestas se generaron y el promedio de aportación y respuesta por alumno. Se observa que en los dos primeros foros hubo mayor promedio de respuestas a los comentarios hechos por los alumnos con 10.3 y 8.3 , al contrario, en los foros 3 y 4 hubo menos respuesta por alumno con 3.0 y 2.2. Estos datos subrayan un nivel muy pequeño de actividad en participar juntos en estos foros, aunque agregaron comentarios de manera normal. 
Tabla N.. 3: Total de aportaciones y respuestas generadas por foro, y promedio por alumno y por foro

\begin{tabular}{|c|c|c|c|c|}
\hline \multirow{2}{*}{ N=23 } & \multicolumn{2}{|c|}{ Temas agregados } & \multicolumn{2}{c|}{ Respuestas } \\
\cline { 2 - 5 } & $\begin{array}{c}\text { Total por } \\
\text { foro }\end{array}$ & $\begin{array}{c}\text { Promedio } \\
\text { por } \\
\text { estudiante }\end{array}$ & $\begin{array}{c}\text { Total por } \\
\text { foro }\end{array}$ & $\begin{array}{c}\text { Promedio } \\
\text { por } \\
\text { estudiante }\end{array}$ \\
\hline Foro 1 & 43 & 1.9 & 236 & 10.3 \\
\hline Foro 2 & 57 & 2.5 & 191 & 8.3 \\
\hline Foro 3 & 45 & 2.0 & 70 & 3.0 \\
\hline Foro 4 & 32 & 1.4 & 51 & 2.2 \\
\hline Foro 5 & 21 & 0.9 & 97 & 4.2 \\
\hline Foro 6 & 23 & 1.0 & 121 & 5.3 \\
\hline Total & 221 & - & 766 & - \\
\hline Promedio & 37 & 1.6 & 127 & 5.5 \\
\hline
\end{tabular}

Por otro lado, si se toma en cuenta que en Moodle se necesitan dos clics tanto para leer un comentario en un foro como para agregar un nuevo tema o una repuesta, los datos en las tablas 2 y 3 , informan que, en promedio, cada estudiante, en cada foro, ha leído 41 comentarios, más o menos dos comentarios de cada otro estudiante, y ha agregado \pm 1.5 nuevos temas y \pm 5.5 respuestas, sea siete intervenciones, lo que implica, más o menos, una actividad de agregación por cada seis comentarios leídos o, visto con un ángulo diferente, la puesta en comunicación de cada estudiante con seis de sus colegas. Estos datos también confirman que los dos primeros foros fueron los que tuvieron mayor participación tanto al nivel de los temas agregados como al de las respuestas.

En la figura $N$ ․ํ 1, ilustrando la distribución de los clics en porcentaje según las rúbricas de Moodle, se observa una disminución constante, de foro en foro, con un pequeño aumento en los dos últimos foros, los con trabajo en equipo, en casi todas la rúbricas. 
Figura N ․ํ 1: Distribución en porcentaje de los clics según las rubricas de Moodle

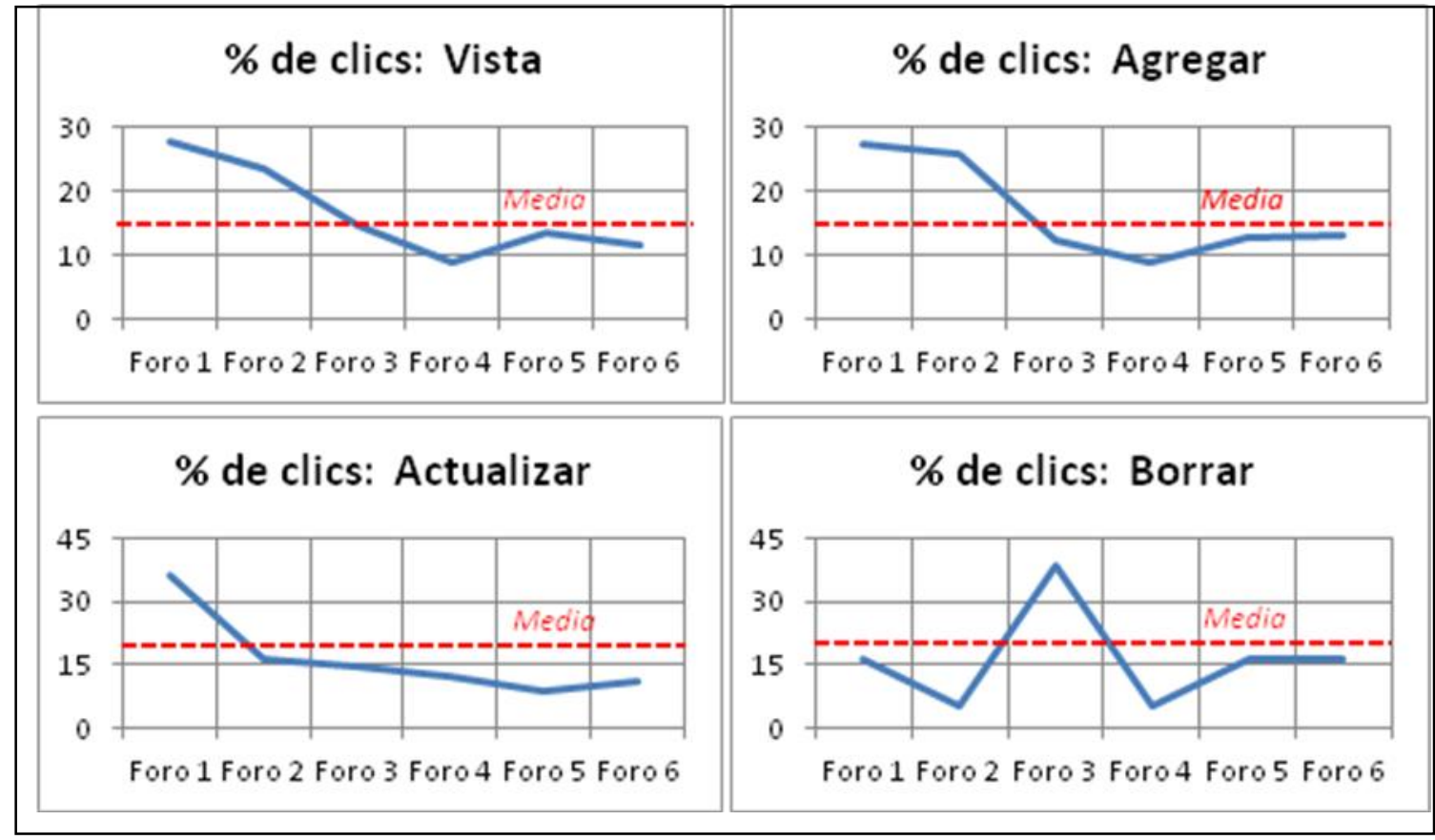

Quizás se pueden explicar las diferencias entre los foros por un menor interés tanto para los temas, Planeación estratégica y prospectiva, como para la fórmula del trabajo, investigar en Internet 3 sitios acerca del tema. -Cabe recordar que la obligación de participar era agregar temas-. Pero pueden, también, explicarse las diferencias de otra manera, la distribución porcentual puede indicar el surgimiento de una lasitud frente a la magnitud y la repetición de los foros y no el desinterés para los últimos temas.

\section{Discusión y conclusión}

Dentro del ambiente virtual de aprendizaje es importante mencionar la utilidad del foro, como una de las herramientas más importantes para el proceso de aprendizaje y colaboración. La importancia de la participación en los foros, que en los resultados se muestra fue tal que trabajar codo a codo a través de esta herramienta, ha contribuido posiblemente no solo al aprendizaje, sino también a la consolidación de relaciones entre los compañeros. Es decir, tal vez el trabajo colaborativo ha buscado la mejora de las relaciones sociales (Pujolas, 2004; Crook, 1998). 
Tomando en cuenta el tamaño relativo del número de clics asociados con los foros, se puede afirmar que el objetivo general del curso se pudo lograr con el uso de los foros para propiciar el aprendizaje colaborativo. De hecho, cerca del $50 \%$ de todos los clics se vinculan con los foros; además, la relación entre las actividades de participación, es decir, agregar, actualizar, borrar comentarios, de un lado, y ver y agregar temas de discusión, por otro, subrayan la interacción sostenida por la tecnología y el intercambio de ideas entre los participantes. En efecto, en la opinión de los estudiantes, el espacio donde más trabajaron colaborativamente fue en los foros de tipo académico. Además, fue evidente que los alumnos en su mayoría seguían un mismo patrón que consistía, en primer lugar, en dar un vistazo de lo que ya habían escrito otros compañeros para analizar la línea a seguir en el tema y después de hacer esta revisión general. Los alumnos se dedicaban a contestar las aportaciones realizadas, o bien, ingresar un nuevo tema de discusión.

Finalmente, los registros de navegación muestran cómo utilizaron los alumnos los foros en Moodle y los resultados obtenidos del análisis de los logs son positivos. La estructura de las actividades en los foros, las cuales son claras y eficaces, abarca el conjunto de posibilidades de participaciones y como Moodle da acceso a los logs de cada actividad se muestra que los alumnos, después de dar una revisión general al enfoque que guiaba el foro, se dedicaban a agregar comentarios nuevos así como respuestas hechas a comentarios de otros alumnos. Es decir, el aprendizaje colaborativo que se originaba en los foros en su mayoría se gestaba de manera dinámica, proporcionando información que generaba debate hasta llegar a una conclusión y así generar posiblemente un aprendizaje.

El detalle de la información transmitida por los registros y la multiplicidad de los tipos de información disponibles son asombrosas, lo que se puede medir en un ambiente virtual de aprendizaje son los intercambios, las interacciones entre estudiantes, pero no el aprendizaje mismo. Se sabe, también, que es difícil identificar cuál es el contenido de un aprendizaje construido por un individuo, delimitar lo que proviene de conocimientos anteriores y entender cómo y con qué se construyeron los nuevos conocimientos. Por último, la noción en sí misma de aprendizaje colaborativo se plantea como limitante al no existir una definición homogénea. Es solamente con el testimonio de los estudiantes a través de encuestas o de grupos de discusión que se puede confirmar que se realizó un aprendizaje de tipo colaborativo, eso sin saber exactamente cual aprendizaje. 
Una limitación tiene que ver con la amplitud del estudio mismo. Participaron solamente 23 estudiantes de una misma carrera y cohorte, eso al nivel del posgrado. Significa que los resultados no se pueden generalizar para cubrir el fenómeno del aprendizaje colaborativo al nivel del posgrado, menos al nivel de la licenciatura. Se necesitan otros estudios, con varias materias y disciplinas, para saber qué pasa exactamente con el aprendizaje colaborativo en la formación universitaria. Sería juicioso, en investigaciones futuras, analizar todas las actividades tanto en los foros como en las otras actividades de cada estudiante y, en el caso de los foros, hasta el contenido de las intervenciones en sí mismas para proponer un perfil personalizado de cada estudiante al nivel de comportamiento de aprendizaje.

Siendo que este estudio se focalizó en el intercambio de información entre estudiantes en un ámbito virtual de aprendizaje, es importante mencionar que la organización de los foros jugó un papel primordial en los resultados de esta investigación. Como, en promedio, cada estudiante se conectó 80 veces con una duración de 31:30 minutos cada vez, el equivalente de 42 horas para el curso completo, y como la duración pro medio de un clic fue de 1 minuto 35 segundos (Díaz, 2010), se debe considerar que cada estudiante tomó 14:19:36 horas para participar en los foros académicos obligatorios, lo que representa bastante tiempo. Eso es una información que los diseñadores de cursos en línea o híbridos deberían tomar en cuenta.

\section{Referencias}

Alvarez, Isabel, Ayuste, Ana, Gros Salvat, Begoña, Guerra, Vania, \& Romañá Blay, María Teresa. (2005). Construir conocimiento con soporte tecnológico para un aprendizaje colaborativo. Revista Iberoamericana de Educación, 36(1), 1-15. Recuperado de http://www.rieoei.org/deloslectores/1058alvarez.pdf

Baker, Ryan. S.J.D., y Yacef, Kalina. (2011). The State of Educational Data Mining in 2009: A Review and Future Visions. Journal of Educational Data Mining, 1(1), 3-17. Recuperado

de: http://www.educationaldatamining.org/JEDM/images/articles/vol1/issue1/JEDMVol1/ssu e1 BakerYacef.pdf

Barros, Beatriz, y Verdejo, Felisa, M. (2001). Entornos para la realización de actividades de aprendizaje colaborativo a distancia. Revista Iberoamericana de Inteligencia Artificial, 5(12), 39-49. Recuperado de: http://www.informatik.unitrier.de/ ley/db/journals/aepia/aepia5.html 
Bartolomé, Antonio. (2002). Universidades en la Red. ¿Universidad presencial o virtual? Critica, LII (896), 34-38. Recuperado de http://www.Imi.ub.es/personal/bartolome/articuloshtml/bartolomeSPcritica02.pdf

Bernaza, Guillermo, y Lee, Francisco. (2005). El aprendizaje colaborativo, una vía para la educación de posgrado. Revista Iberoamericana de Educación, 37(3), 1-18. Recuperado de www.rieoei.org/1123.htm

Brito, Vivina. (marzo, 2004). El foro electrónico: una herramienta tecnológica para facilitar el aprendizaje colaborativo. Edutec. Revista Electrónica de Tecnología Educativa. 17, 1-20. Recuperado de: http://dialnet.unirioja.es/servlet/articulo?codigo $=926914$

Cabrera, Elsa P. (2004). Aprendizaje colaborativo soportado por computador: su estado actual. Revista Iberoamericana de Educación, 33(6), 1-16. Recuperado de: http://www.rieoei.org/deloslectores/729Cabrera108.PDF

Calzadilla, María Eugenia. (2002). Aprendizaje colaborativo y tecnologías de la información y la comunicación. Revista Iberoamericana de educación, 1-11. Recuperado de: www.rieoei.org/tec edu7.htm

Crook, Charles. (1998). Ordenadores y aprendizaje colaborativo. Madrid: Ediciones Morata.

Dewey, John. (1989). Como pensamos: Nueva exposición de la relación entre pensamiento y proceso educativo. Madrid: Paidós.

Díaz, Karla. M. (2010). Relación entre estilos de aprendizaje y navegación de un curso en línea de la Maestría en Ciencias Educativas de la UABC. Tesis de Maestría no publicada. Ensenada: Instituto de Investigación y Desarrollo Educativo, Universidad Autónoma de Baja California, México.

Dönmez, Pinar, Rosé, Carolyn, Stegmann, Karsten, Weinberger, Armin y Fischer, Frank. (2005). Supporting CSCL with automatic corpus analysis technology. Proceedings of the International Conference of Computer Support for Collaborative Learning (CSCL 2005), 125-134. http://www.cs.cmu.edu/ cprose/pubweb/donmez05.pdf

Dringus, Laurie, P., Ellis, Timothy. (2005). Using data mining as a strategy for assessing asynchronous discussion forums, Computer and Education Journal, 45, 141-160.

Duart, Josep M., Gil, Marc, Pujol, Maria y Castaño, Jonatan. (2008). La universidad en la sociedad red. Barcelona: Ariel.

Escribano González, Alicia. (1995). Aprendizaje cooperativo y autónomo en la enseñanza universitaria. Enseñanza \& Teaching: Revista interuniversitaria de didáctica 13, 89104. Recuperado de http://espacio.uned.es/fez/eserv.php?pid=bibliuned:20478\&dsID=aprendizaje cooperativo.pdf 
Garcia, Francisco Jesus. (2000). Accesibilidad, educación y tecnologías de la información $y$ la comunicación. Recuperado de http://ares.cnice.mec.es/informes/17/versionpdf.pdf

Garrido, Antoni. (2003). El aprendizaje como identidad de participación en la práctica de una comunidad virtual. Recuperado de http://www.uoc.edu/in3/dt/20088/index.html

Glinz Férez, Patricia Elizabeth. (2005). Un acercamiento al trabajo colaborativo. Revista Iberoamericana de Educación, s.n., 1-14. Recuperado de http://www.rieoei.org/deloslectores/820Glinz.PDF

Hoskins, Sherria L. y van Hooff, Johanna C. (2005). Motivation and ability: which students use online learning and what influence does it have on their achievement? British Journal of Educational Technology, $36 \quad$ (2), 177-192. Recuperado de http://onlinelibrary.wiley.com/doi/10.1111/bjet.2005.36.issue-2/issuetoc

International Working Group on Educational Data Mining (IWGEDM). (2011). Events. Recuperado de http://www.educationaldatamining.org/

Johnson, David, W. \& Johnson, Roger T. (1989) Cooperation and competition: Theory and Research. Edina, MN: Interaction Book Co.

Lavigne, Gilles, Backhoff, Eduardo E., Organista, Javier S. (2008) La hibridación digital del proceso educativo. En Javier Vales Garcia (Ed.) Nuevas Tecnologías para el Aprendizaje. (43-60) México, D.F.: Pearson-Prentice Hall.

Lavigne, Gilles, Organista, Javier S. y McAnally Lewis S. (2008). Étude préliminaire des pratiques de navigation dans un environnement. Revue internationale des technologies en pédagogie universitaire, 5(1), 6-26. Recuperado de: http://www.ritpu.org/IMG/pdf/RITPU v05n01 06.pdf

Lavigne, Gilles, McAnally, Lewis S., Organista, Javier, S., Días, Karla M. L. y Vasconcelos, Pricila M. O. (2011). Moodle et ses registres de navigation: pouvons-nous en tirer des connaissances pratiques? (pp. 10-16). Congrès de la Association Francophone International de Recherche Scientifique en Éducation (AFIRSE), UNESCO, Paris, France.

Liponen, Lasse, Hakkarainen, Kai, y Paavola, Sami. (2004). Practices and orientations of CSCL. En Strijbos, Jan-Willem, Kirschner, Paul A., y Martens, Rob L. (Eds.), What we know about CSCL (31-46). Norwell (MA): Kluwer Academic Publisher.

Martí, Eduardo. (1992). Aprender con ordenadores en la escuela. Barcelona: Horsori Editorial.

Muhlenbrock, Martin. (1999). A system for analyzing collaborative problem solving. Recuperado de http://citeseerx.ist.psu.edu/viewdoc/summary?doi=10.1.1.25.8372 
Perera, Dilhan, Kay, Judy, Koprinska, I., Yacef, Kalina y Zaiane, Osmar. (2009) Clustering and sequential pattern mining to support team learning. IEEE Transactions on Knowledge and Data Engineering 21-6, 759-772 Recuperado de http://sydney.edu.au/engineering/it/ irena/tkde.pdf

Polly, Jean Armour. (1992, junio). Surfing the Internet. Wilson Library Bulletin 66(10) 38-42, ERIC document EJ447456.

Pujolas, Pere Maset. (2004). Contribución de las TIC al aprendizaje cooperativo. 4as. Jornadas: Las Tecnologías de la Información y la Comunicación. Internet en el centro educativo, Zaragoza, Es. Recuperado de http://recursos.cepindalo.es/mod/resource/view.php?id=9647

Ríos, Pablo. (2005). La mediación del aprendizaje. Cuadernos UCAB (1) Lev Vygotsky, aportes para el siglo XXI (pp. 34-40). Caracas: Publicaciones UCAB.

Sacristán Gimeno, José \& Pérez Gomez, Angel. (1994). Comprender y transformar la enseñanza. Barcelona: Morata.

Serrano, José Manuel y Calvo, Maria Teresa. (1994). Aprendizaje cooperativo. Técnicas y análisis dimensional. Murcia: Obra Cultural de Cajamurcia.

SOLAR. (2011). Open Learning Analytics. Solar's Concept Paper. Recuperado de http://solaresearch.org/OpenLearningAnalytics.pdf

Vasconcelos, Pricila M. O. (2011) Aprendizaje Colaborativo en un ambiente virtual: Estudio preliminar. Tesis de Maestría no publicada, Ensenada: Instituto de Investigación y Desarrollo Educativo, Universidad Autónoma de Baja California, México.

Vygotsky, Lev. (2005). Pensamiento y Lenguaje. México: Quinto Sol. 